\title{
Precision Standard Model Measurements at the Tevatron
}

\author{
Tommaso Dorigo \\ for the $\mathrm{CDF}$ and $\mathrm{D} \varnothing$ collaborations \\ Dipartimento di Fisica “G.Galilei”, Via Marzolo 8, 35131 Padova, Italy
}

\begin{abstract}
.
The CDF and DØ collaborations at the Tevatron have produced exquisite precision measurements on high- $P_{T}$ physics with their large datasets of $p \bar{p}$ collisions. The top quark is being studied in great detail, and a precision of $1.1 \%$ in the measurement of its mass has been achieved. The large datasets of $W$ and $Z$ boson decays have allowed the most precise measurement of the $W$ mass to date, and detailed studies of production and decay asymmetries; moreover, associated production of pairs of vector bosons have been observed and measured. The precise knowledge of top and $W$ masses are providing decisive new input for the allowed mass range of a standard model Higgs boson, as well as for the parameter space of benchmark scenarios in supersymmetric theories.
\end{abstract}

Keywords: Standard Model, top quark, Higgs boson, electroweak production, hadron collisions PACS: $12.38 . \mathrm{Qk}, 13.85 . \mathrm{Qk}, 13.85 . \mathrm{Rm}, 14.65 . \mathrm{Ha}, 14.80 . \mathrm{Bn}, 14.80 . \mathrm{pp}$

\section{THE FACILITIES}

The Tevatron is the highest energy collider in the world, producing proton-antiproton interactions at a center-of-mass energy of $1.96 \mathrm{TeV}$. The collider underwent a massive upgrade at the turn of the millennium, with the construction of a new injector and several improvements in the facility producing and storing antiprotons, which constitute the most challenging part of the project. A peak luminosity of $2.9 \times 10^{32} \mathrm{~cm}^{-2} \mathrm{~s}^{-1}$ has been recently surpassed, and the integrated luminosity of $3.2 \mathrm{fb}^{-1}$ has been delivered so far to the $\mathrm{CDF}$ and $\mathrm{D} \varnothing$ experiments. That number is expected to at least double by the end of 2009.

An overview of the CDF and DØ detectors for Run II (2001-present) can be found in[1]. In what follows the most important features for standard model tests in high- $P_{T}$ processes are briefly mentioned.

Both detectors are all-purpose, near-hermetic devices consisting of a tracker immersed in a solenoidal field and an outer shell of calorimeters and muon chambers. In D $\varnothing$ an excellent system of silicon microstrips has been installed in Run II. Six barrels of silicon sensors organized in four concentrical layers provide coverage for central tracks, while a total of sixteen silicon disks allow recostruction of large rapidity tracks. A similar set of seven barrels of silicon strips is organized in the core of CDF.

Outside the silicon barrels CDF features a large gas tracking chamber, while $\mathrm{D} \varnothing$ has a compact scintillating fiber tracker. Central tracks are reconstructed with very high efficiency in both detectors, and the combination with silicon detector information allows the determination of track impact parameter with sufficient precision to reconstruct B- 

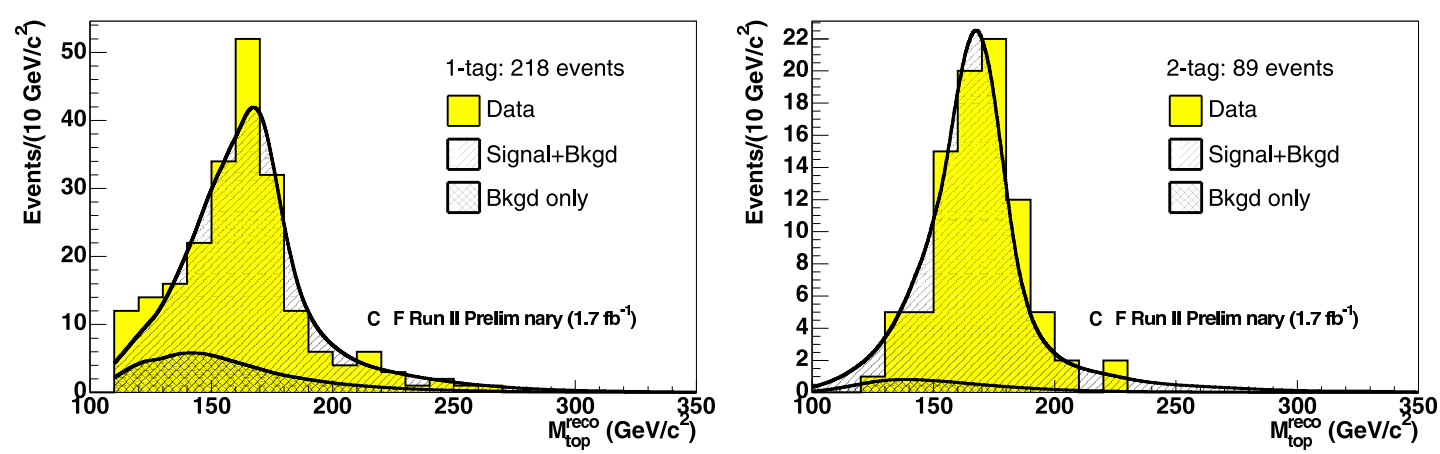

FIGURE 1. Distribution of the reconstructed top mass from events with one (left) or two (right) $b$ tagged jets (yellow histogram), with an overlaid fit to signal and background templates (black line).

hadron decay and enable $\sim 45 \%$-efficient tagging of $b$-quark-originated jets, with fake rates well below $1 \%$.

Calorimeters are finely segmented in projective towers and are divided in a inner electromagnetic and an outer hadronic section. Electrons within the pseudorapidity interval $|\eta|<2.0$ are identified with high purity and efficiency, with a resolution of about $15 \% / \sqrt{E}$ in both detectors, and hadronic jets are reconstructed with resolutions better than $100 \% / \sqrt{E}$. Muon chambers cover the rapidity region $|\eta|<1.5$ in CDF and $|\eta|<2.0$ in D $\varnothing$

Data acquisition proceeds through a three-level trigger system which is capable of reducing the $2.5 \mathrm{MHz}$ collision rate by selecting online the most interesting events, writing full detector information to tape at the rate of about $100 \mathrm{~Hz}$. Most of the high$P_{T}$ measurements discussed in this paper use datasets collected by triggers identifying isolated electrons and muons.

\section{TOP QUARK MEASUREMENTS}

The heaviest quark is a particularly interesting laboratory for perturbative QCD, given its large mass and short lifetime. The large datasets collected by CDF and D $\varnothing$ enable many precision measurements of top quark properties. Among them, the top mass $M_{t}$ is the most urgent to determine precisely, since it represents a crucial input to the global fits to electroweak observables which verify the internal consistency of the theory and provide a prediction for the unknown mass of the Higgs boson.

There are by now tens of determinations of $M_{t}$, using different techniques and all the significant final states of top decay. The most recent precise result from CDF is based on $1.7 \mathrm{fb}^{-1}$ of data in the single-lepton final state of top pair production. 307 events are selected to contain a high- $P_{T}$ lepton, missing transverse energy, and four jets, at least one of which is tagged to originate from $b$-quark hadronization. The dijet mass from the hadronic $W$ boson decay is used to reduce the uncertainty arising from the jet energy scale, using two-dimensional probability density functions to compare the reconstructed top quark mass and hadronic $W$ mass distributions in data and simulations. 

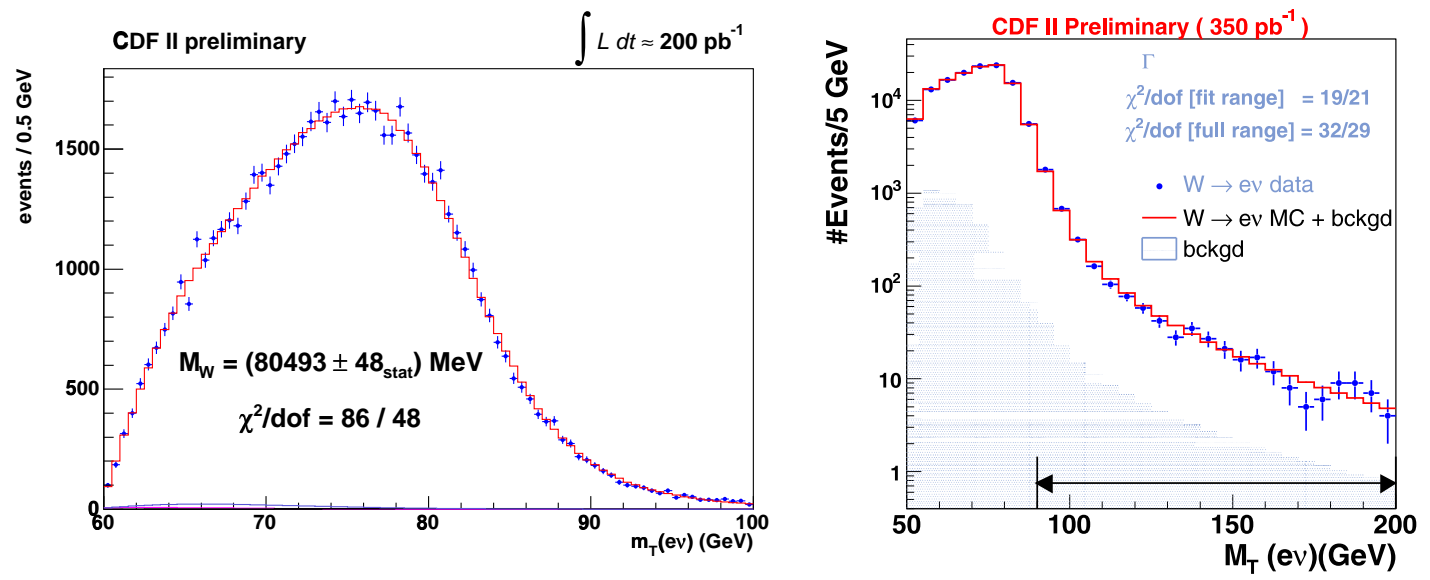

FIGURE 2. Left: the $W$ transverse mass distribution computed by CDF from electron-neutrino pairs (blue points) and the result of a likelihood fit (red histogram). Right: a fit to the tail of the $\mathrm{W}$ transverse mass distribution for muon-neutrino pairs allows the extraction of the natural width of the $W$ boson.

The top mass and the jet energy scale are thus determined simultaneously. CDF finds $M_{t}=171.6 \pm 2.1 \pm 1.1 \mathrm{GeV}$, where the first error is the statistical plus energy scale uncertainty, and the second accounts for other systematics (see figure 1).

Recent results from $\mathrm{D} \varnothing$ include a measurement performed in the very clean sample of dilepton decays of top quark pairs, which consists of 57 candidates in $1 \mathrm{fb}^{-1}$. Two different weighting methods are used to extract a top mass from the unconstrained final state, which includes two undetectable neutrinos from the leptonic $W$ decays. The combination of the two results yields $M_{t}=173.7 \pm 5.4$ (stat.) \pm 3.4 (syst.) GeV.

The latest average of CDF and D $\varnothing$ dterminations of $M_{t}$ is based on datasets including less than $1 \mathrm{fb}^{-1}$ of data. The top mass is measured at $M_{t}=170.9 \pm 1.1 \pm 1.5 \mathrm{GeV}$. Before the end of Run II the two experiments are likely to reach a $1 \mathrm{GeV}$ precision on the top quark mass.

The search for electroweak production of single top quarks is still ongoing. The combined cross section of the contributing processes is only two times smaller than that of QCD pair production, but the signal has a much less characteristic signature, and is buried in the large background of QCD $W+$ jets events. Both $\mathrm{D} \varnothing$ and CDF have seen evidence for single top events in their data, with statistical significances exceeding the level of $3 \sigma$. A definitive observation will most likely be achieved next year.

\section{VECTOR BOSON PHYSICS}

The samples of leptonic $W$ and $Z$ boson decays collected by CDF and D $\varnothing$ now count millions of events. These datasets have allowed very precise measurements of electroweak processes and observables, including the world's most precise value for the $W$ boson mass by $\operatorname{CDF}\left(M_{W}=80413 \pm 48 \mathrm{MeV}\right)$, which is the result of a long and detailed analysis of only $200 \mathrm{pb}^{-1}$ of electron and muon data (see figure 2, left). By the end of Run 

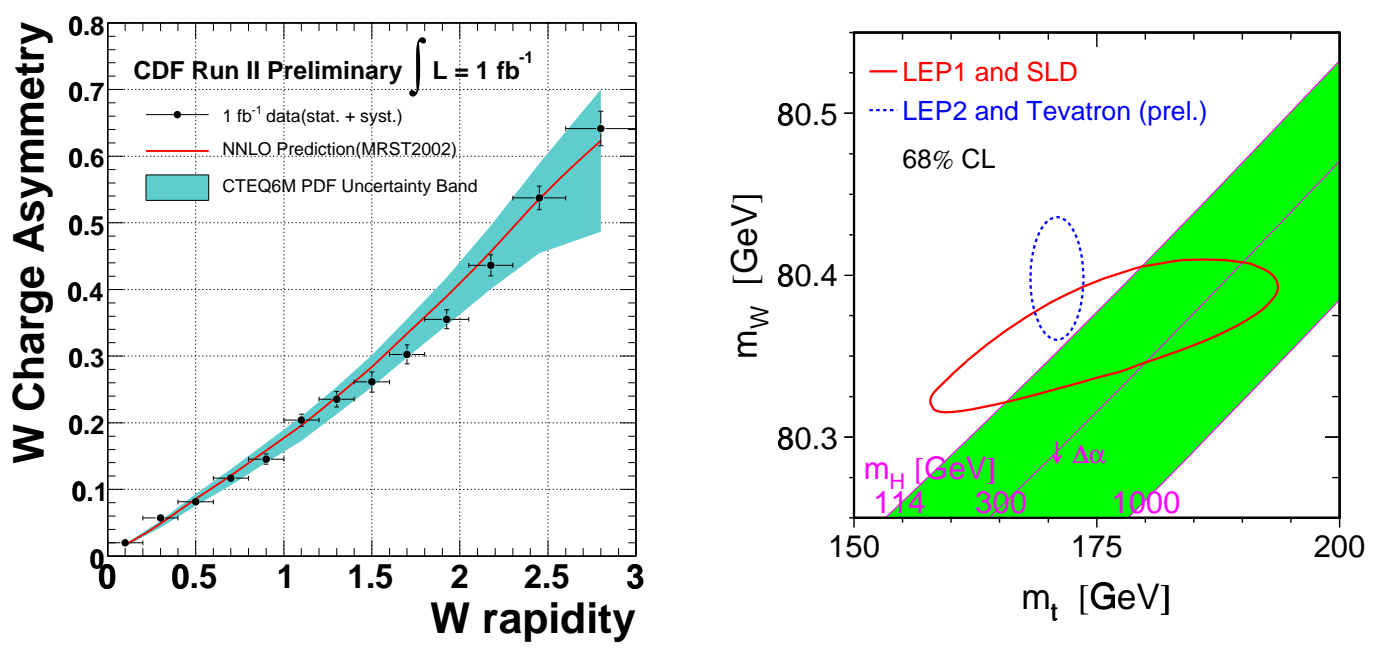

FIGURE 3. Left: the $\mathrm{W}$ charge asymmetry measured by CDF as a function of $W$ boson rapidity (black points), compared to next-to-next-to leading order predictions (red line) and the uncertainty of the CTEQ6M parton distribution functions (blue band). Right: comparison of direct measurments of top and $W$ masses (blue ellipse) with indirect constraints from LEP (red ellipse). The green band shows the expected dependence of the two parameters in the standard model, for a wide range of Higgs boson masses.

II the Tevatron experiments expect to reduce the uncertainty on $M_{W}$ below $20 \mathrm{MeV}$.

Another quantity measured with high precision is the natural width of the $W$ boson, which is determined with a fit to the tail of the transverse mass distribution of lepton and missing energy. Again CDF has the most precise measurement of this quantity: $\Gamma_{W}=2032 \pm 71 \mathrm{MeV}$, in good agreement with standard model predictions (see figure 2, right). CDF and D $\varnothing$ have also produced several measurements of cross sections and asymmetries in vector boson production. The left plot in figure 3 shows a recent determination of the $W$ charge asymmetry in CDF, which provides a stringent constraint to the parton distribution functions of the proton at medium $x$.

Very rare electroweak processes have also started to emerge: all associated production processes involving pairs of $W$ and $Z$ bosons have now been detected, with cross sections in excellent agreement with theoretical predictions. These signatures are studied in detail given the importance of multi-lepton signals for searches of the Higgs boson and new physics. Table 1 lists the Run II results obtained by CDF and DØ on diboson cross sections.

\section{IMPLICATIONS}

Global fits to electroweak observables in the context of the standard model have recently started to point to a very small value of the Higgs boson mass. This trend is largely due to the precise measurements of the top quark and $W$ boson masses obtained at the Tevatron (see figure 3, right). The most recent fits predict a Higgs mass $M_{H}=76_{-24}^{+33} \mathrm{GeV}$, well 
TABLE 1. Measured inclusive cross sections for diboson production processes at the Tevatron. The column labeled "NLO" shows the standard model prediction computed at next-to leading order; when multiple uncertainties are quoted in the CDF and $\mathrm{D} \varnothing$ measurements, they refer in order to statistical, systematic, and luminosity errors.

\begin{tabular}{lccc}
\hline & NLO pred. & CDF & D \\
\hline$\sigma_{p \bar{p} \rightarrow W W+X}$ & $12.4 \pm 0.8 \mathrm{pb}$ & $13.6 \pm 2.3 \pm 1.6 \pm 1.2 \mathrm{pb}$ & $13.8_{-3.8-0.9}^{+4.3+1.2} \pm 0.9 \mathrm{pb}$ \\
$\sigma_{p \bar{p} \rightarrow W Z+X}$ & $3.7 \pm 0.3 \mathrm{pb}$ & $4.3_{-1.0}^{+1.3} \pm 0.2 \pm 0.3 \mathrm{pb}$ & $2.7_{-1.3}^{+1.7} \mathrm{pb}$ \\
$\sigma_{p \bar{p} \rightarrow Z Z+X}$ & $1.4 \pm 0.1 \mathrm{pb}$ & $0.75_{-0.54}^{+0.71} \mathrm{pb}$ & - \\
\hline
\end{tabular}

* J.M.Campbell and R.K.Ellis, Phys. Rev. D60 (1999) 113006.

below the lower experimental bound set by the LEP II experiments; the upper limit on $M_{H}$ has been set at $144 \mathrm{GeV}$, at $95 \%$ confidence level[2].

If one examines the contribution of each input measurement to the above result one notices that the strongest downward pulls on $M_{H}$ are due to the lepton asymmetry measurements by SLD[3] and the world average value of $M_{W}\left(M_{W}=80398 \pm 25 \mathrm{MeV}\right)$. There is some tension in the mixture of results, but the fit is reasonably good: the standard model still holds, although in the next few years the increased precision of the Tevatron measurements -especially on $M_{W}$ - could strain it to the breaking point. On the other hand, in the context of minimal supersymmetric models the overall agreement of all observables appears very good for a wide chunk of the parameter space of a few benchmark scenarios. In particular, within the NUHM (a non-universal Higgs model), it has been shown that one can fit well also B-physics and dark matter constraints to the lightest supersymmetric particle, obtaining a coherent picture[4]. Many options are still on the table, but the ongoing searches for the Higgs boson at the Tevatron, and those due to start soon at the LHC, are likely to clarify matters.

\section{CONCLUSIONS}

The Tevatron experiments are producing exquisite new results on high- $P_{T}$ physics and are bringing standard model tests to a level of precision which meets or exceeds that of electron-positron colliders. The top quark mass is now known with $1.1 \%$ precision, and the $W$ boson mass with $0.04 \%$ precision. These results are contributing to narrower margins of consistency of global fits to electroweak observables. CDF and D $\varnothing$ will continue to produce excellent physics through 2009 , and possibly after that. They expect to reach a $0.7 \%$ precision on the top mass and a $0.02 \%$ precision on the $W$ mass.

\section{REFERENCES}

1. T. Lecompte and H.T. Diehl, The CDF and DØ upgrades for Run II, Ann. Rev. Nucl. Part. Sci 50 (2000) 71.

2. The LEP Electroweak Workin Group, http://lepewwg.web.cern.ch/LEPEWWG/ .

3. R.S.Panvini, Int. J. Mod. Phys. A16 S1A (2001) 292.

4. J. Ellis, S. Heinemeyer, K.A. Olive, A.M. Weber, and G. Weiglein, Hep-ph/07060652. 